\title{
STABILITY OF LARGE-SCALE SYSTEMS WITH LAGGED INTERCONNECTIONS
}

\author{
P. E. KLOEDEN
}

(Received 25 June 1979)

(Revised 12 November 1979)

\begin{abstract}
The aggregation-decomposition method is used to derive sufficient conditions for the uniform stability, uniform asymptotic stability and exponential stability of the null solution of large-scale systems described by functional differential equations with lags appearing only in the interconnections. The free subsystems are described by ordinary differential equations for which converse theorems involving Lyapunov functions exist and thus enable the sufficient conditions to be expressed in terms of Lyapunov functions rather than the more complicated Lyapunov functionals.
\end{abstract}

\section{Introduction}

The aggregation-decomposition method is an effective way of determining stability properties of certain classes of dynamical systems with non-linearities and high dimensions (see, for example, $[1,3,5])$. Basically, it involves the decomposition of a complicated system into several simpler subsystems, each a function of different components of the state vector, with interconnections between them. These subsystems may have some physical meaning or may be just mathematical artifices. Moreover, they need not all be stable for the overall system to be stable. The sum of their more easily found Lyapunov functions is tried as a Lyapunov function for some desired stability property of the overall system. Its suitability is, however, not tested directly, but rather is determined by the negative definiteness or negative semi-definiteness of an aggregation matrix, the elements of which are determined by the interconnections and the Lyapunov functions of the subsystems. This method has been applied to systems described by equations of diverse forms, including ordinary differential equations, difference equations, sampled data ordinary differential equations and functional differential equations. With systems 
described by functional differential equations it is usual to consider a function space as the state space of the system and to derive Lyapunov stability conditions in terms of Lyapunov functionals rather than simpler Lyapunov functions (see, for example, $[3,5])$. The reason for this is that converse stability theorems involving Lyapunov functions do not exist for such systems.

In this paper, the systems considered are described by functional differential equations which have the time lags occurring only in the interconnections and, for these systems, the free subsystems are thus described by ordinary differential equations. Converse stability theorems guarantee the existence of Lyapunov functions for such free subsystems, and the sum of these Lyapunov functions provides a Lyapunov function, rather than a Lyapunov functional, for the overall system even though the overall system is described by a functional differential equation. In Section 2 a detailed description is given of these systems and in Section 3 sufficient conditions involving Lyapunov functions are given for the uniform stability, uniform asymptotic stability and exponential stability of the null solution of a system described by a functional differential equation. The conditions for the first two of these are due to Razumikhin $[6,7]$ and those for exponential stability, which do not seem to have appeared elsewhere, are derived here. In Section 4 the main results involving the aggregation-decomposition method and Lyapunov functions are derived. These give sufficient conditions for the uniform stability, uniform asymptotic stability and exponential stability of the null solution of the overall system. These conditions complement those given in $[3,5]$, and may in some instances be simpler to use. Their application is illustrated with two simple examples in Section 5.

\section{System description}

A system $S$ to be considered is described by an $n$-dimensional functional differential equation (see, for example, Hale [2])

$$
\frac{d x}{d t}=f\left(t, x_{t}\right)
$$

where $x_{t} \in C=C\left([-h, 0], \mathbf{R}^{n}\right)$, and can be decomposed into $s$ interconnected systems $S_{i}$ described by $n_{i}$-dimensional $\left(\sum_{i=1}^{s} n_{i}=n\right)$ functional differential equations

$$
\frac{d x_{i}}{d t}=g_{t}\left(t, x_{i}\right)+h_{i}\left(t, x_{t}\right), \quad i=1,2, \ldots, s .
$$

The $n_{i}$-dimensional functionals $h_{i}: \mathbf{R} \times C \rightarrow \mathbf{R}^{n_{i}}$ in (2) are called interconnections and the vectors $x_{i} \in \mathbf{R}^{n_{i}}$ of the $s$ subsystems $S_{i}$ partition the vector $x \in \mathbf{R}^{n}$, that is, 
$x=\left(x_{1}, x_{2}, \ldots, x_{s}\right)$. It is assumed that $f: \mathbf{R} \times C \rightarrow \mathbf{R}^{n}$ and the $g_{i}: \mathbf{R} \times \mathbf{R}^{n_{t}} \rightarrow \mathbf{R}^{n_{t}}$, $i=1,2, \ldots, s$, are sufficiently smooth to ensure global existence and future continuation of all solutions $x_{t}\left(t_{0}, \phi_{0}\right) \in C$ of system $S$ and all solutions $x_{i}\left(t ; t_{0}, \phi_{0}\right) \in \mathbf{R}^{n_{i}}$ of the free subsystem $S_{i}^{*}$ described by the $n_{i}$-dimensional ordinary differential equation

$$
\frac{d x_{i}}{d t}=g_{i}\left(t, x_{i}\right)
$$

for $i=1,2, \ldots, s$. Further, it is assumed that $g_{i}\left(t, x_{i}\right)=0$ for all $t$ if $x_{i}=0$ for $i=1,2, \ldots, s$, and that $f\left(t, x_{t}\right)=0$ and $h_{i}\left(t, x_{t}\right)=0, i=1,2, \ldots, s$, for all $t$ if $x_{t}=0$.

For $i=1,2, \ldots, s$ an arbitrary norm $\left\|x_{i}\right\|_{i}$ will be used on $\mathbf{R}^{n_{i}}$, but for convenience the max norm,

$$
\|x\|=\max \left\{\left\|x_{i}\right\|_{i} ; i=1,2, \ldots, s\right\},
$$

will be used on $\mathbf{R}^{n}$. Similarly, the max norm,

$$
\|\phi\|_{0}=\max \{\|\phi(u)\| ;-h \leqslant u \leqslant 0\},
$$

will be used on the function space $C$.

In the sequel it is supposed that associated with each free subsystem $S_{i}^{*}$, $i=1,2, \ldots, s$, is a differentiable Lyapunov function $V_{i}\left(t, x_{i}\right)$ which is defined for all $\left(t, x_{i}\right) \in \mathbf{R} \times \mathbf{R}^{n_{i}}$ and satisfies

$$
a_{i}\left(\left\|x_{i}\right\|_{i}\right) \leqslant V_{i}\left(t, x_{i}\right) \leqslant b_{i}\left(\left\|x_{i}\right\|_{i}\right)
$$

for all $(t, x) \in \mathbf{R} \times \mathbf{R}^{n_{i}}$, where $a_{i}(r)$ and $b_{i}(r)$ are continuous, strictly increasing, positive functions of $r \in \mathbf{R}^{+}$with $a_{i}(0)=b_{i}(0)=0$ and $a_{i}(r) \rightarrow \infty$ as $r \rightarrow \infty$; the Lyapunov function also satisfies

$$
\begin{aligned}
\left.\frac{d}{d t} V_{i}\left(t, x_{i}\right)\right|_{3} & =\frac{\partial}{\partial t} V_{i}\left(t, x_{i}\right)\left[\operatorname{grad} V_{i}\left(t, x_{i}\right)\right]^{\mathrm{T}} g_{i}\left(t, x_{i}\right) \\
& \leqslant \mu_{i} c_{i}\left(\left\|x_{i}\right\|_{i}\right)
\end{aligned}
$$

for all $\left(t, x_{i}\right) \in \mathbf{R} \times \mathbf{R}^{n_{i}}$, where $\mu_{i}=1,0$ or -1 and $c_{i}(r)$ is a continuous, strictly increasing, positive function of $r \in \mathbf{R}^{+}$with $c_{i}(0)=0$. For $\mu_{i}=-1$ such a Lyapunov function exists when the null solution of the free subsystem $S_{i}^{*}$ given by (3) is uniformly asymptotically stable; for $\mu_{i}=0$ when it is uniformly stable, and for $\mu_{i}=+1$ when it is unstable in a certain way. See Yoshizawa [8, Sections 18 and 19]. In addition it is assumed that the $s$ interconnection functionals

satisfy

$$
h_{i}\left(t, x_{t}\right), \quad i=1,2, \ldots, s,
$$

$$
\sum_{i=1}^{s}\left[\operatorname{grad} V_{i}\left(t, \phi_{i}(0)\right)\right]^{\mathrm{T}} h_{i}(t, \phi) \leqslant \sum_{i, j=1}^{s} \alpha_{i j} \sqrt{ }\left(d_{i}\left(\left\|\phi_{i}(0)\right\|_{i}\right) d_{j}\left(\left\|\phi_{j}(0)\right\|_{j}\right)\right)
$$


for all $(t, \phi) \in \mathbf{R} \times C$, where the $\alpha_{i j}$ are real numbers with $\alpha_{i j}=\alpha_{j i}$ and the $s$ functions $d_{i}(r)$ are continuous, strictly increasing, positive functions of $r \in \mathbf{R}^{+}$ with $d_{i}(0)=0$ for $i=1,2, \ldots, s$.

Alternatively, in the sequel it is supposed that associated with each free subsystem $S_{i}^{*}, i=1,2, \ldots, s$, is a differentiable Lyapunov function $V_{i}\left(t, x_{i}\right)$ which is defined for all $\left(t, x_{i}\right) \in \mathbf{R} \times \mathbf{R}^{n_{t}}$ and satisfies

$$
a_{i}\left\|x_{i}\right\|_{i} \leqslant V_{i}\left(t, x_{i}\right) \leqslant b_{i}\left\|x_{i}\right\|_{i}
$$

and

$$
\left.\frac{d}{d t} V_{i}\left(t, x_{i}\right)\right|_{3} \leqslant \mu_{i} c_{i}\left\|x_{i}\right\|_{i}
$$

for all $\left(t, x_{i}\right) \in \mathbf{R} \times \mathbf{R}^{n_{i}}$, where $a_{i}, b_{i}$ and $c_{i}$ are positive constants and $\mu_{i}=-1,0$ or +1 . For $\mu_{i}=-1$ such Lyapunov functions exist when the null solution of the free subsystem $S_{i}^{*}$ given by (3) is exponentially stable; for $\mu_{i}=0$ when it is uniformly stable, and for $\mu_{i}=+1$ when it is unstable in a certain way. Finally, in this alternative case, it is also assumed that the $s$ interconnection functionals $h_{i}\left(t, x_{t}\right), i=1,2, \ldots, s$, satisfy

$$
\sum_{i=1}^{s}\left[\operatorname{grad} V_{i}\left(t, \phi_{i}(0)\right)\right]^{\mathrm{T}} h_{i}(t, \phi) \leqslant \sum_{i, j=1}^{s} \alpha_{i j} \sqrt{ }\left(V_{i}\left(t, \phi_{i}(0)\right) V_{j}\left(t, \phi_{j}(0)\right)\right)
$$

for all $(t, \phi) \in \mathbf{R} \times C$, where the $\alpha_{i j}$ are real numbers with $\alpha_{i j}=\alpha_{j i}$.

\section{Lyapunov functions for functional differential equations}

For a dynamical system $S$ described by a functional differential equation (1), it is now common practice to consider the function space $C$ as the state space of the system rather than the euclidean space $\mathbf{R}^{n}$ (see, for example, $[2,4,8]$ ). The reason for this is that much of the well-developed theory of dynamical systems described by ordinary differential equations, in particular the semigroup evolution property and Lyapunov stability theory, then translates almost directly into this function space setting. For instance, definitions of stability, asymptotic stability and exponential stability of the null solution $x_{t}=0$ of a system $S$ given by (1) become:

DEFINITION. The null solution $x_{t}=0$ of a system $S$ given by (1) is stable if, for every $t_{0} \in \mathbf{R}$ and $\varepsilon>0$, there exists a $\delta=\delta\left(t_{0}, \varepsilon\right)>0$ such that

$$
\left\|x_{t}\left(t_{0}, \phi_{0}\right)\right\|_{0}<\varepsilon
$$

for all $t \geqslant t_{0}$ and all $\phi_{0} \in C$ with $\left\|\phi_{0}\right\|_{0}<\delta$. It is asymptotically stable if, in addition, there exists $a \delta_{0}=\delta_{0}\left(t_{0}\right)>0$ such that

$$
\left\|x_{t}\left(t_{0}, \phi_{0}\right)\right\|_{0} \rightarrow 0
$$


as $t \rightarrow \infty$ for all $\phi_{0} \in C$ with $\left\|\phi_{0}\right\|_{0}<\delta_{0}$. These stabilities are uniform if $\delta$ and $\delta_{0}$ are independent of $t_{0}$.

The null solution $x_{t}=0$ of a system $S$ given by (1) is exponentially stable if there exist positive constants $K$ and $c$ such that

$$
\left\|x_{t}\left(t_{0}, \phi_{0}\right)\right\|_{0} \leqslant K\left\|\phi_{0}\right\|_{0} e^{-c\left(t-t_{0}\right)}
$$

for all $t \geqslant t_{0}$ and all $\phi_{0} \in C$.

With a function state space, Lyapunov stability conditions are expressed in terms of Lyapunov functionals $V\left(t, x_{t}\right)$ defined on $\mathbf{R} \times C$. Both necessary and sufficient conditions involving Lyapunov functionals have been derived for the above stabilities (see, for example, $[2,4,8]$ ).

The main advantage of considering the euclidean space $\mathbf{R}^{n}$ as the state space of a system $S$ described by a functional differential equation (1) is that euclidean state vectors are in practice easier to work with than are state functions. In particular, Lyapunov stability conditions can then be expressed in terms of Lyapunov functions $V(t, x)$ rather than the more complicated Lyapunov functionals. Unfortunately, in contrast with Lyapunov functionals, the existence of Lyapunov functions is in general not guaranteed for the above stabilities, that is, necessary conditions involving Lyapunov functions cannot, in general, be derived (see Hale [2, page 221]). Sufficient conditions can, however, be derived. The following sufficient conditions for uniform stability and uniform asymptotic stability of the null solution of a system $S$ given by (1) are due originally to Razumikhin [6, 7]. See also Lakshmikantham and Leela [4, chapter 8].

THEOREM 1. Suppose there exists a differentiable function $V: \mathbf{R} \times \mathbf{R}^{n} \rightarrow \mathbf{R}$ such that

$$
a(\|x\|) \leqslant V(t, x) \leqslant b(\|x\|)
$$

for all $(t, x) \in \mathbf{R} \times \mathbf{R}^{n}$, where $a(r)$ and $b(r)$ are continuous, strictly increasing, positive functions of $r \in \mathbf{R}^{+}$with $a(0)=b(0)=0$ and $a(r) \rightarrow \infty$ as $r \rightarrow \infty$; suppose further that $V$ is such that

$$
\begin{aligned}
\left.\frac{d V}{d t}\right|_{1} & =\frac{\partial}{\partial t} V(t, \phi(0))+[\operatorname{grad} V(t, \phi(0))]^{\mathrm{T}} f(t, \phi) \\
& \leqslant \mu c(\|\phi(0)\|)
\end{aligned}
$$

for all $(t, \phi) \in \mathbf{R} \times C$, where $\mu$ is a constant and $c(r)$ is a continuous, strictly increasing, positive function of $r \in \mathbf{R}^{+}$with $c(0)=0$.

Then, if $\mu=0$, the null solution $x_{t}=0$ of a system $S$ given by (1) is uniformly stable and, if $\mu<0$, it is uniformly asymptotically stable. 
The following sufficient conditions involving a Lyapunov function hold for the exponential stability of the null solution of a system $S$ given by (1). A proof is included here as none seems to have appeared elsewhere.

THEOREM 2. Suppose there exists a differentiable function $V: \mathbf{R} \times \mathbf{R}^{n} \rightarrow \mathbf{R}$ such that

$$
a\|x\| \leqslant V(t, x) \leqslant b\|x\|
$$

for all $(t, x) \in \mathbf{R} \times \mathbf{R}^{n}$, where $a$ and $b$ are positive constants; suppose further that $V$ is such that

$$
\left.\frac{d V}{d t}\right|_{1} \leqslant \mu c V(t, \phi(0))
$$

for all $(t, \phi) \in \mathbf{R} \times C$, where $c$ is a positive constant and $\mu$ is a constant.

Then, if $\mu=0$, the null solution $x_{t}=0$ of a system $S$ given by (1) is uniformly stable and, if $\mu<0$, it is exponentially stable.

Proof. The case $\mu=0$ follows from the previous theorem. For the case $\mu<0$ it can be assumed without loss of generality that $\mu=-1$. Then, by Theorem 8.1.4 of Lakshmikantham and Leela [4] with $g\left(t, r, r_{t}\right)=-c r$, by the right-hand inequality of (14), and by (15), it follows that

$$
\begin{aligned}
V\left(t, x\left(t ; t_{0}, \phi_{0}\right)\right) & \leqslant V\left(t_{0}, \phi_{0}(0)\right) e^{-c\left(t-t_{0}\right)} \\
& \leqslant b\left\|\phi_{0}(0)\right\| e^{-c\left(t-t_{0}\right)} \\
& \leqslant b\left\|\phi_{0}\right\|_{0} e^{-c\left(t-t_{0}\right)}
\end{aligned}
$$

for all $t \geqslant t_{0}$ and all $\phi_{0} \in C$. Hence, by the left-hand inequality of (14),

$$
a\left\|x\left(t ; t_{0}, \phi_{0}\right)\right\| \leqslant b\left\|\phi_{0}\right\|_{0} e^{-c\left(t-t_{0}\right)},
$$

that is,

$$
\left\|x\left(t ; t_{0}, \phi_{0}\right)\right\| \leqslant b a^{-1}\left\|\phi_{0}\right\|_{0} e^{-c\left(t-t_{0}\right)},
$$

for all $t \geqslant t_{0}$ and all $\phi_{0} \in C$. Since $b a^{-1} \geqslant 1$, it thus follows that

$$
\left\|x_{t}\left(t, \phi_{0}\right)\right\|_{0} \leqslant b a^{-1}\left\|\phi_{0}\right\|_{0} e^{-c\left(t-t_{0}\right)}
$$

for all $t \geqslant t_{0}$ and all $\phi_{0} \in C$, which proves the theorem.

\section{Main results}

In view of there being no necessary conditions to guarantee their existence, Lyapunov functions are, in general, not as useful a means as Lyapunov functionals 
of investigating the stability of a system described by a functional differential equation (1). This situation is, however, reversed when the functional differential equation can be decomposed into the form (2), because then the required Lyapunov functions are provided by the free subsystems $S_{i}^{*}$, which are described by ordinary differential equations (3), for which necessary conditions do exist.

When the free subsystems $S_{i}^{*}$ have Lyapunov functions $\dot{V}_{i}\left(t, x_{i}\right), i=1,2, \ldots, s$, satisfying conditions (6) and (7) and when the interconnections satisfy (8), a real, symmetric, $s \times s$ matrix $A=\left(a_{i j}\right)$, called an aggregation matrix and defined by

$$
a_{i j}=\mu_{i} \delta_{i j}+\alpha_{i j}, \quad i, j=1,2, \ldots, s,
$$

where $\delta_{i j}$ is the Kronecker delta symbol, is formed and the function

$$
V(t, x)=\sum_{i=1}^{s} V_{i}\left(t, x_{i}\right)
$$

is tried as a Lyapunov function for the uniform stability or the uniform asymptotic stability of the null solution of the system $S$ given by (1). The suitability of this function need not, however, be tested directly. Instead, it is determined by whether $A$ is a negative semi-definite or a negative definite matrix.

\section{THEOREM 3. Suppose that}

(a) the Lyapunov function $V_{i}\left(t, x_{i}\right)$ of the free subsystem $S_{i}^{*}$ given by (3) satisfies (6) and (7) for $i=1,2, \ldots, s$;

(b) the interconnection functionals $h_{i}\left(t, x_{t}\right), i=1,2, \ldots, s$, satisfy (8);

(c) the aggregation matrix $A=\left(a_{i j}\right)$ defined by (16) is negative definite.

Then the null solution $x_{t}=0$ of the system $S$ given by (1) is uniformly asymptotically stable. If the aggregation matrix $A$ is only negative semi-definite, then the null solution is uniformly stable.

Proof. The theorem will be proved by showing that the function $V(t, x)$ defined by (17) satisfies conditions (12) and (13) of Theorem 1 .

The function $V(t, x)$ is defined and differentiable for all $(t, x) \in \mathbf{R} \times \mathbf{R}^{n}$ on account of (17) and the global definition and differentiability of the $s$ functions $V_{i}\left(t, x_{i}\right)$, $i=1,2, \ldots, s$.

From the properties of the $s$ functions $a_{i}(r), i=1,2, \ldots, s$, the function

$$
a(r)=\min \left\{a_{i}(r) ; i=1,2, \ldots, s\right\}
$$

is a continuous, strictly increasing, positive function of $r \in \mathbf{R}^{+}$with $a(0)=0$ and $a(r) \rightarrow \infty$ as $r \rightarrow \infty$. From definition (4) of the max norm and from the left-hand inequality in (6), for each $(t, x) \in \mathbf{R} \times \mathbf{R}^{n}$ there is a $j=1,2, \ldots, s$, which depends on $x$, such that $\|x\|=\left\|x_{j}\right\|_{j}$. Consequently,

$$
a(\|x\|) \leqslant a_{j}\left(\left\|x_{j}\right\|_{j}\right) \leqslant V_{j}\left(t, x_{j}\right) \leqslant V(t, x),
$$


that is, for all $(t, x) \in \mathbf{R} \times \mathbf{R}^{n}$,

$$
a(\|x\|) \leqslant V(t, x) .
$$

Similarly, from the properties of the $s$ functions $b_{i}(r)$, the function

$$
b(r)=\max \left\{s b_{i}(r) ; i=1,2, \ldots, s\right\}
$$

is a continuous, strictly increasing, positive function of $r \in \mathbf{R}^{+}$with $b(0)=0$. From (4) and the right-hand inequality in (6),

$$
\begin{aligned}
V(t, x) & =\sum_{i=1}^{s} V_{i}\left(t, x_{i}\right) \\
& \leqslant \sum_{i=1}^{s} b_{i}\left(\left\|x_{i}\right\|_{i}\right) \\
& \leqslant \sum_{i=1}^{s} b_{i}(\|x\|) \leqslant b(\|x\|)
\end{aligned}
$$

for all $(t, x) \in \mathbf{R} \times \mathbf{R}^{n}$.

Along the solutions of the system $S$ given by (1) the derivative of $V(t, x)$ is, for any $(t, \phi) \in \mathbf{R} \times C$,

$$
\begin{aligned}
\left.\frac{d V}{d t}\right|_{1} & =\frac{\partial}{\partial t} V(t, \phi(0))+[\operatorname{grad} V(t, \phi(0))]^{\mathrm{T}} f(t, \phi) \\
& =\sum_{i=1}^{s} \frac{\partial}{\partial t} V_{i}\left(t, \phi_{i}(0)\right)+\left[\operatorname{grad} V_{i}\left(t, \phi_{i}(0)\right)\right]^{\mathrm{T}}\left(g_{i}\left(t, \phi_{i}(0)\right)+h_{i}(t, \phi)\right) \\
& =\left.\sum_{i=1}^{s} \frac{d V_{i}}{d t}\right|_{3}+\sum_{i=1}^{s}\left[\operatorname{grad} V_{i}\left(t, \phi_{i}(0)\right)\right]^{\mathrm{T}} h_{i}(t, \phi) \\
& \leqslant \sum_{i=1}^{s} \mu_{i} c_{i}\left(\left\|\phi_{i}(0)\right\|_{i}\right)+\sum_{i, j=1}^{s} \alpha_{i j} \sqrt{ }\left(d_{i}\left(\left\|\phi_{i}(0)\right\|_{i}\right) d_{j}\left(\left\|\phi_{j}(0)\right\|_{j}\right)\right) \\
& \leqslant\left(\sqrt{ } e_{1}, \sqrt{ } e_{2}, \ldots, \sqrt{ } e_{s}\right) A\left(\sqrt{ } e_{1}, \sqrt{ } e_{2}, \ldots, \sqrt{ } e_{s}\right)^{\mathrm{T}} \\
& \leqslant \lambda_{\max }(A) \sum_{i=1}^{s} e_{i}\left(\left\|\phi_{i}(0)\right\|_{i}\right) \\
& \leqslant-\left|\lambda_{\max }(A)\right| c(\|\phi(0)\|),
\end{aligned}
$$

where $\lambda_{\max }(A)<0$ is the largest eigenvalue of the negative definite aggregation matrix $A$, the $s$ functions $e_{i}(r)$ are defined by

$$
e_{i}(r)=\max \left\{c_{i}(r), d_{i}(r)\right\}
$$

for $i=1,2, \ldots, s$, and the function $c(r)$ is defined by

$$
c(r)=\max \left\{s e_{i}(r) ; i=1,2, \ldots, s\right\} .
$$


From the properties of the $2 s$ functions $c_{i}(r)$ and $d_{i}(r), i=1,2, \ldots, s$, it is clear that the function $c(r)$ is a continuous, strictly increasing, positive function of $r \in \mathbf{R}^{+}$with $c(0)=0$.

Hence the Lyapunov function $V(t, x)$ satisfies the sufficient conditions (12) and (13) of Theorem 1 with $\mu=-1$ and thus the null solution $x_{t}=0$ of the system $S$ given by (1) is uniformly asymptotically stable.

If the aggregation matrix $A$ is negative semi-definite, then its largest eigenvalue $\lambda_{\max }(A) \leqslant 0$. Hence the Lyapunov function $V(t, x)$ satisfies the sufficient conditions (12) and (13) of Theorem 1 with $\mu=0$ and thus the null solution $x_{t}=0$ of the system $S$ given by (1) is uniformly stable.

This completes the proof of the theorem.

When the free subsystems $S_{i}^{*}$ have Lyapunov functions $V_{i}\left(t, x_{i}\right), i=1,2, \ldots, s$, satisfying conditions (9) and (10) with the interconnections satisfying condition (11), the aggregation matrix $A=\left(a_{i j}\right)$ is defined by

$$
a_{i j}=\mu_{i} c_{i} \delta_{i}+\alpha_{i j}, \quad i, j=1,2, \ldots, s .
$$

The function $V(t, x)$ defined by (17) is then tried as a Lyapunov function to test for the exponential stability or the uniform stability of the null solution of the system $S$ given by (1). As previously, the former holds if the aggregation matrix $A$ defined by (18) is negative definite and the latter if it is negative semi-definite.

\section{THEOREM 4. Suppose that}

(a) the Lyapunov function $V_{i}\left(t, x_{i}\right)$ of the free subsystem $S_{i}^{*}$ given by (3) satisfies (9) and (10) for $i=1,2, \ldots, s$;

(b) the interconnection functionals $h_{i}\left(t, x_{t}\right), i=1,2, \ldots, s$, satisfy $(11)$;

(c) the aggregation matrix $A=\left(a_{i j}\right)$ defined by (18) is negative definite.

Then the null solution $x_{t}=0$ of the system $S$ given by (1) is exponentially stable. If the aggregation matrix $A$ is only negative semi-definite, then the null solution is uniformly stable.

Proof. The theorem will be proved by showing that the function $V(t, x)$ defined by (17) satisfies conditions (14) and (15) of Theorem 2 .

As in the previous theorem, $V(t, x)$ is defined and differentiable for all $(t, x) \in \mathbf{R} \times \mathbf{R}^{n}$. It also satisfies

$$
a\|x\| \leqslant V(t, x) \leqslant b\|x\|
$$

for all $(t, x) \in \mathbf{R} \times \mathbf{R}^{n}$, where

$$
a=\min \left\{a_{i} ; i=1,2, \ldots, s\right\} \text { and } b=\max \left\{s b_{i} ; i=1,2, \ldots, s\right\} .
$$


Along the solutions of the system $S$ given by (1) the derivative of $V(t, x)$ is, for all $(t, \phi) \in \mathbf{R} \times C$,

$$
\begin{aligned}
\left.\frac{d V}{d t}\right|_{1} & =\left.\sum_{i=1}^{s} \frac{d V_{i}}{d t}\right|_{3}+\sum_{i=1}^{s}\left[\operatorname{grad} V_{i}\left(t, \phi_{i}(0)\right)\right]^{\mathrm{T}} h_{i}(t, \phi) \\
& \leqslant \sum_{i=1}^{s} \mu_{i} c_{i} V_{i}\left(t, \phi_{i}(0)\right)+\sum_{i, j=1} \alpha_{i j} \sqrt{ }\left(V_{i}\left(t, \phi_{i}(0)\right) V_{j}\left(t, \phi_{j}(0)\right)\right) \\
& \leqslant\left(v_{1}, v_{2}, \ldots, v_{s}\right) A\left(v_{1}, v_{2}, \ldots, v_{s}\right)^{\mathrm{T}} \\
& \leqslant \lambda_{\max }(A) \sum_{i=1}^{s} V_{i}\left(t, \phi_{i}(0)\right) \\
& =-\left|\lambda_{\max }(A)\right| V(t, \phi(0)),
\end{aligned}
$$

where $\lambda_{\max }(A)<0$ is the largest eigenvalue of the negative definite aggregation $A$ and $v_{1}=\sqrt{ }\left(V_{i}\left(t, \phi_{i}(0)\right)\right)$ for $i=1,2, \ldots, s$.

Hence the Lyapunov function $V(t, x)$ satisfies the sufficient conditions (14) and (15) of Theorem 2 with $\mu=-1$ and $c=\left|\lambda_{\max }(A)\right|$, and thus the null solution $x_{t}=0$ of the system $S$ given by (1) is exponentially stable.

When the aggregation matrix $A$ is negative semi-definite, then its largest eigenvalue $\lambda_{\max }(A) \leqslant 0$. Hence the Lyapunov function $V(t, x)$ satisfies the sufficient conditions (14) and (15) of Theorem 2 with $\mu=0$, and thus the null solution $x_{i}=0$ of the system $S$ given by (1) is uniformly stable.

This completes the proof of the theorem.

\section{Examples}

The following two simple examples illustrate the application of Theorems 3 and 4 above. In each example there are two one-dimensional free subsystems, described by ordinary differential equations, for which the null solutions are either exponentially stable or unstable.

EXAMPLE 1. The system $S$ is composed of two interconnected one-dimensional subsystems described by the functional differential equations

$$
\left.\begin{array}{rl}
\frac{d x_{1}}{d t} & =-x_{1}(t)+h_{1}\left(t, x_{t}\right), \\
\frac{d x_{2}}{d t} & =x_{2}(t)+h_{2}\left(t, x_{t}\right),
\end{array}\right\}
$$

where

$$
h_{1}\left(t, x_{t}\right)=x_{1}(t)\left|x_{2}(t)\right|
$$


and

$$
h_{2}\left(t, x_{t}\right)=-x_{2}(t) \int_{-h}^{0}\left\{x_{1}^{2}(t+u)+2 x_{1}(t+u)+2\right\} d u .
$$

The null solution $x_{1}=0$ of the free subsystem $S_{1}^{*}$ described by the ordinary differential equation

$$
\frac{d x_{1}}{d t}=-x_{1}
$$

is exponentially stable. A suitable Lyapunov function is

$$
V_{1}\left(x_{1}\right)=x_{1}^{2},
$$

for which

$$
\left.\frac{d V_{1}}{d t}\right|_{20}=-2 x_{1}^{2}
$$

for all $x_{1} \in \mathbf{R}$. Conditions (6) and (7) are thus satisfied with $a_{1}(r)=b_{1}(r)=r$, $c_{1}(r)=2 r^{2}$ and $\mu_{1}=-1$.

The null solutions $x_{2}=0$ of the free subsystem $S_{2}^{*}$ described by the ordinary differential equation

$$
\frac{d x_{2}}{d t}=x_{2}
$$

is unstable. A suitable Lyapunov function is

for which

$$
V_{2}\left(x_{2}\right)=x_{2}^{2}
$$

$$
\left.\frac{d V_{2}}{d t}\right|_{21}=2 x_{2}^{2}
$$

for all $x_{2} \in \mathbf{R}$, and so conditions (6) and (7) are satisfied with $a_{2}(r)=b_{2}(r)=r^{2}$, $c_{2}(r)=2 r^{2}$ and $\mu_{2}=+1$.

The bounds (8) for these Lyapunov functions and the interconnection functionals are determined as follows:

$$
\left[\operatorname{grad} V_{1}\left(\phi_{1}(0)\right)\right] h_{1}(t, \phi)=\phi_{1}^{2}(0)\left|\phi_{2}(0)\right|
$$

and

$$
\begin{aligned}
{\left[\operatorname{grad} V_{2}\left(\phi_{2}(0)\right)\right] h_{2}(t, \phi) } & =-2 \phi_{2}^{2}(0) \int_{-h}^{0}\left\{\phi_{1}^{2}(u)+2 \phi_{1}(u)+2\right\} d u \\
& \leqslant 2 \phi_{2}^{2}(0) \int_{-h}^{0}(-1) d u \\
& \leqslant-2 h \phi_{2}^{2}(0)
\end{aligned}
$$


for all $\phi \in C$. Hence

$$
\sum_{i=1}^{2}\left[\operatorname{grad} V_{i}\left(\phi_{i}(0)\right) h_{i}(t, \phi) \leqslant \phi_{1}^{2}(0)\left|\phi_{2}(0)\right|-2 h \phi_{2}^{2}(0)\right.
$$

for all $\phi \in C$, and so condition (8) is satisfied with

$$
\alpha_{11}=0, \quad \alpha_{12}=\alpha_{21}=1, \quad \alpha_{22}=-2 h, \quad d_{1}(r)=r^{4} \quad \text { and } \quad d_{2}(r)=r^{2}
$$

But

$$
\mu_{1}=-1 \text { and } \mu_{2}=+1 \text {, }
$$

and so the aggregation matrix defined by (16) is

$$
A=\left[\begin{array}{cc}
-1 & 1 \\
1 & 1-2 h
\end{array}\right]
$$

This is negative definite for $h>1$ and negative semi-definite for $h=1$. Hence, by Theorem 3, the null solution $x_{t}=0$ of the system $S$ given by (19) is uniformly asymptotically stable if the delay $h>1$, and uniformly stable if the delay $h=1$.

EXAMPLE 2. The system $S$ is composed of two interconnected one-dimensional subsystems described by the functional differential equations

$$
\left.\begin{array}{l}
\frac{d x_{1}}{d t}=2 x_{1}(t)+h_{1}\left(t, \dot{x}_{t}\right), \\
\frac{d x_{2}}{d t}=-2 x_{2}(t)+h_{2}\left(t, x_{t}\right),
\end{array}\right\}
$$

where

$$
h_{1}\left(t, x_{t}\right)=x_{2}(t) \operatorname{sgn}\left(x_{1}(t) x_{2}(t)\right)-3 x_{1}(t) \cosh \left(\int_{-h}^{0} x_{2}(t+u) d u\right)
$$

and

$$
h_{2}\left(t, x_{t}\right)=x_{1}(t) \operatorname{sgn}\left(x_{1}(t) x_{2}(t)\right) .
$$

The null solution $x_{1}=0$ of the free subsystem $S_{1}^{*}$ described by the ordinary differential equation

$$
\frac{d x_{1}}{d t}=2 x_{1}
$$

is unstable. A suitable Lyapunov function is

for which

$$
V_{1}\left(x_{1}\right)=x_{1}^{2}
$$

$$
\left.\frac{d V_{1}}{d t}\right|_{23}=4 x_{1}^{2}=4 V_{1}\left(x_{1}\right)
$$


for all $x_{1} \in \mathbf{R}$, and so conditions (9) and (10) are satisfied with $a_{1}(t)=b_{1}(r)=r^{2}$, $c_{1}=4$ and $\mu_{1}=+1$.

The null solution $x_{2}=0$ of the free subsystem $S_{2}^{*}$ described by the ordinary differential equation

$$
\frac{d x_{2}}{d t}=-2 x_{2}
$$

is exponentially stable. A suitable Lyapunov function is

$$
V_{2}\left(x_{2}\right)=x_{2}^{2}
$$

for which

$$
\left.\frac{d V_{2}}{d t}\right|_{24}=-4 x_{2}^{2}=-4 V_{2}\left(x_{2}\right)
$$

for all $x_{2} \in \mathbf{R}$, and so conditions (9) and (10) are satisfied with $a_{2}(r)=b_{2}(r)=r^{2}$, $c_{2}=4$ and $\mu_{2}=-4$.

The bounds (11) and these Lyapunov functions and the interconnection functionals are determined as follows:

$\left[\operatorname{grad} V_{1}\left(\phi_{1}(0)\right)\right] h_{1}(t, \phi)=2 \phi_{1}(0) \phi_{2}(0) \operatorname{sgn}\left(\phi_{1}(0) \phi_{2}(0)\right)$

$$
-6 \phi_{1}^{2}(0) \cosh \left(\int_{-h}^{0} \phi_{2}(u) d u\right)
$$

$$
\begin{aligned}
& \leqslant 2\left|\phi_{1}(0) \phi_{2}(0)\right|-6 \phi_{1}^{2}(0) \\
& =2 \sqrt{ }\left(V_{1}\left(\phi_{1}(0)\right) V_{2}\left(\phi_{2}(0)\right)\right)-6 V_{1}\left(\phi_{1}(0)\right)
\end{aligned}
$$

for all $\phi \in C$. Hence condition (11) is satisfied with $\alpha_{11}=-6, \alpha_{12}=\alpha_{21}=1$ and $\alpha_{22}=0$. But $c_{1}=c_{2}=4, \mu_{1}=+1$ and $\mu_{2}=-1$, and so the aggregation matrix defined by (18) is

$$
A=\left[\begin{array}{cc}
-2 & 1 \\
1 & -4
\end{array}\right]
$$

This is negative definite with eigenvalues $(-3 \pm \sqrt{ } 5)<0$. Hence the null solution $x_{t}=0$ of the system $S$ given by (22) is exponentially stable.

\section{References}

[1] L. T. Grujić and D. D. Siljak, "Asymptotic stability and instability in large-scale systems", IEEE Trans. Auto. Control 18 (1973), 636-645.

[2] J. K. Hale, Theory of functional differential equations (Springer, New York, 1977). 
[3] G. S. Ladde, "Stability in large-scale hereditary systems under structural perturbations", Proc. IFAC Symp. on Large-scale Systems Theory and Applics, Udine, Italy, June 16-20, 1976, pp. 215-226.

[4] V. Lakshmikantham and S. Leela, Differential and integral inequalities, Vol. 2 (Academic Press, New York, 1971).

[5] A. N. Michel and R. K. Miller, Qualitative analysis of large scale dynamical systems (Academic Press, New York).

[6] B. S. Razumikhin, "Stability of systems with lag", Prikl. Matem. Mekh. 20 (1956), 500-512 (in Russian).

[7] B. S. Razumikhin, “Application of Lyapunov's method to problems in the stability of systems with a delay", Automat. i Telemekh. 21 (1960), 740-748.(in Russian).

[8] T. Yoshizawa, Stability theory by Lyapunov's second method (Math. Soc. Japan, Tokyo, 1966).

School of Mathematical and Physical Sciences

Murdoch University

Murdoch

Western Australia 6153 